\title{
OTIMIZAÇÃO DA PROGRAMAÇÃO DO ABASTECIMENTO DE CRUS EM REFINARIAS DE PETRÓLEO
}

\author{
V. V. Viero ${ }^{1,2}$ e S. M. S. Neiro ${ }^{1,3}$ \\ ${ }^{1}$ Universidade Federal de Uberlândia, Faculdade de Engenharia Química \\ E-mail para contato: ${ }^{2}$ vini.vidoto@gmail.com ; ${ }^{3}$ sergioneiro@feq.ufu.br
}

\begin{abstract}
RESUMO - O objetivo do presente trabalho é desenvolver um modelo de otimização que contemple o problema de abastecimento de crus às refinarias. Para isto foi utilizada uma adaptação do modelo proposto por Hu e Zhu (2007) e foram obtidos bons resultados computacionais, além de ter sido possível modelar demandas operacionais essenciais.
\end{abstract}

\section{INTRODUÇÃO}

Vive-se em uma era onde o petróleo é uma das mais importantes commodities de um país, levando a diversas tentativas de melhorarmos os lucros obtidos com a comercialização deste produto. Uma destas tentativas é o estudo da programação da produção das refinarias de petróleo, mas por gerarem problemas de grande dificuldade de resolução ao observar todo o escopo de uma refinaria, dividem-se as atividades da mesma em três partes: descarregamento e mistura de óleos crus, produção de derivados e mistura e envio de produtos, como proposto por Jia e Iearapetritou (2003). Desses três problemas o mais amplamente estudado, e sobre o qual será tratado neste artigo, é a programação da produção do descarregamento e mistura de crus, pois é uma fase critica no processo de refino, já que quando otimizado pode gerar grande economia, como reportado em Chen, Grossmann e Zheng (2012).

A programação da produção é um processo de tomada de decisões de grande importância nas indústrias do segundo e terceiro setores da economia (Pinedo e Chao, 1999). A aplicação de otimização ao problema de programação da produção visa obter uma solução que determine a utilização dos recursos disponíveis atendendo às exigências de demanda $\mathrm{e}$ atendendo às limitações de capacidade, qualidade, entre outras. (Ierapetritou e Floudas, 1998). Em Chen, Grossmann e Zheng (2012) foram revisados 3 modelos considerados estado da arte e um dos modelos mais promissores é o modelo Unit Slot. Portanto, neste trabalho, adotou-se como ponto inicial este modelo proposto por Hu e Zhu (2007), que é um modelo de tempo continuo e cujo foco das equações é nas unidades.

\section{DESCRIÇÃO DO PROBLEMA}

O problema resolvido neste trabalho consiste em uma refinaria fictícia, ilustrada na Figura 1, que recebe 3 volumes de óleo cru por um oleoduto com 6 tanques de 


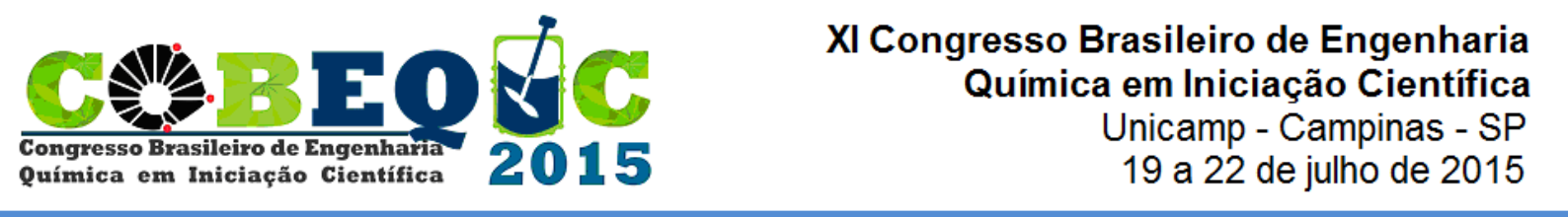

armazenamento e mistura e 2 unidades de destilação (UDCs) e 12 tipos de petróleo cru e nosso objetivo é maximizar o lucro da mesma.

O petróleo chega à refinaria através do oleoduto na forma de parcelas (volumes) de petróleo cru que são descarregadas para os tanques. A mistura de cada tanque é alimentada às unidades de destilação. Até dois tanques podem alimentar uma mesma unidade de destilação ao mesmo tempo, da mesma forma que um tanque pode alimentar até duas UDCs. A imposição de qualidade é feita após a válvula onde há a mistura da alimentação dos tanques associados a uma carga. As decisões do problema de otimização consistem em definir para quais tanques as parcelas serão descarregadas, o gerenciamento dos tipos de cru em cada tanque, quais tanques serão alocados para a alimentação de cada UDC.

\section{MODELAGEM MATEMÁTICA}

O modelo consiste de uma série de equações de balanço de massa e restrições lógicas envolvendo tempo, qualidade de certas propriedades dos crus, entre outras, como vemos abaixo e a não linearidade vem da Equação 21. Um caso de estudo envolvendo uma refinaria próxima da real foi usado para a construção deste modelo atendendo, suas exigências, mas aqui iremos reportar somente as equações que captam a ideia básica da abordagem unit slot.

O problema foi resolvido na plataforma GAMS IDE e foi utilizada uma estratégia de decomposição do problema em dois, para resolvê-lo. Primeiramente foi resolvido como um problema MIP através do método contido no pacote cplex da plataforma, ignorando a equação que gera a não linearidade. Então, as variáveis binárias obtidas na primeira parte da resolução foram fixadas e foi resolvido o problema completo, como um MINLP relaxado com o método do pacote dinopt da mesma plataforma.

\subsection{Nomenclatura}

Conjuntos:

$\begin{array}{ll}\mathbf{i} & \text { Conjunto de priority-slots, sendo i0 o primeiro e n o último; } \\ \mathbf{r}, \mathbf{r} 1 & \text { Conjuntos de todos os recursos; } \\ \mathbf{c} & \text { Conjunto de crus; } \\ \mathbf{k} & \text { Conjunto de propriedades; } \\ \mathbf{r p}(\mathbf{r}) & \text { Conjunto de parcelas; } \\ \mathbf{r t}(\mathbf{r}) & \text { Conjunto de tanques; } \\ \mathbf{r m}(\mathbf{r}) & \text { Conjunto de mixers; } \\ \mathbf{r d}(\mathbf{r}) & \text { Conjunto de unidades de destilação; } \\ \mathbf{i n}(\mathbf{r}, \mathbf{r 1}) & \text { Recurso r1 que abastece o recurso r (entrada em r); } \\ \mathbf{o u t}(\mathbf{r}, \mathbf{r 1}) & \text { Recurso r que abastece o recurso r1 (saída em r); }\end{array}$

$\underline{\text { Constantes: }}$

H Horizonte de scheduling (foi utilizado $\mathrm{H}=144$ );

TR Tempo de preparação (foi utilizado TR $=24$ ); 
Parâmetros:

VTn(r,r1) Volume mínimo de transferência;

VTx(r,r1) Volume máximo de transferência;

$\operatorname{PP}(\mathrm{c}, \mathrm{k})$

$\operatorname{PPn}(k)$

$\operatorname{PPx}(k)$

G(c)

$\operatorname{Tarr}(\mathbf{r})$

Propriedade de cada tipo de petróleo (acidez, densidade, enxofre);

Mínimo valor para a qualidade $\mathrm{k}($ acidez $=0,05$ e enxofre $=0,05)$;

Máximo valor para a qualidade $\mathrm{k}($ acidez $=1,25$ e enxofre $=0,767)$;

Margens de contribuição no lucro;

Chegada de uma parcela;

Tabela 1 - Dados adicionais dos parâmetros

\begin{tabular}{|c|c|c|c|c|c|}
\hline Petróleo & G(c) & Recurso & Tarr & VTn & VTx \\
\hline P1 & 105 & Vol1 & 0 & 5400 & 19500 \\
\hline P2 & 160 & Vol2 & 25 & 5400 & 17500 \\
\hline P3 & 135 & Vo13 & 35 & 5400 & 20000 \\
\hline P4 & 133 & TQ1 & - & 100 & 30000 \\
\hline P5 & 149 & TQ2 & - & 100 & 34000 \\
\hline P6 & 152 & TQ3 & - & 100 & 30000 \\
\hline P7 & 100 & TQ4 & - & 100 & 33000 \\
\hline P8 & 143 & TQ5 & - & 100 & 38000 \\
\hline P9 & 122 & TQ6 & - & 100 & 38000 \\
\hline P10 & 115 & MIX1 & - & 12960 & 76000 \\
\hline P11 & 139 & MIX2 & - & 9072 & 76000 \\
\hline P12 & 129 & & & &
\end{tabular}

Variáveis:

Ganho

Resultado da função objetivo que maximiza o lucro;

Vt(i,r,r1) Volume total de óleo transferido entre os recursos no slot i;

Vc(i,r,r1,c) Volume de crú transferido entre os recursos no slot i;

Lt(i,r)

Lc(i,r,c)

$\mathbf{S}(\mathbf{i}, \mathbf{r})$

$\mathbf{E}(\mathbf{i}, \mathbf{r})$

XT(i,r)

$\mathbf{Z}(\mathbf{i}, \mathbf{r})$

Volume total acumulado de óleo no tanque no início do slot $\mathrm{i}$;

Volume acumulado de crú no tanque no início do slot i;

Momento inicial da operação de transferência entre recursos no slot i;

Momento final da operação de transferência entre recursos no slot i;

Variável que identifica o recebimento de parcelas no tanque de carga;

Variável binaria que indica se há transferência entre recursos no slot i;

\subsection{Modelagem}

Função Objetivo:

- Função Objetivo que visa maximizar o lucro gerado pela destilação.

Ganho $=\sum_{i} \sum_{r d} \sum_{r m \in i n(r d, r m)} \sum_{c} \frac{G(c) * V_{c}(i, r m, r d, c)}{1000}$ 
Restrições lógicas:

- Número máximo de tanques na carga da destilação.

$\sum_{r t \in i n(r m, r t)} Z(i, r t, r m) \leq 2$

- Se houver carga de qualquer parcela em um tanque a variável XT deve ser ativada.

$X T(i, r t) \geq Z(i, r p, r t)$

- Se não houver carga de qualquer parcela em um tanque a variável XT deve ser nula.

$X T(i, r t) \leq \sum_{r p \in i n(r t, r p)} Z(i, r p, r t)$

Restrições de tempo:

- O tempo de cada recurso deve ser monotonicamente crescente.

$S(i, r) \geq E(i-1, r)$

- O fim de uma operação deve ocorrer depois de seu início.

$E(i, r) \geq S(i, r)$

- Relação dos tempos de início entre parcelas e tanques.

$S(i, r t) \leq S(i, r p)+(H-\operatorname{Tarr}(r p)) *(1-Z(i, r p, r t))$

- Relação dos tempos de término entre parcelas e tanques.

$E(i, r t) \leq E(i, r p)-H^{*}(1-Z(i, r p, r t))$

- Relação dos tempos de início entre tanques e mixers.

$S(i, r t) \leq S(i, r m)+H^{*}(1-Z(i, r t, r m))$

- Relação dos tempos de término entre tanques e mixers.

$E(i, r t) \geq E(i, r m)-H^{*}(1-Z(i, r t, r m))$

- Relação dos tempos de início entre mixers e destilação.

$S(i, r m)=S(i, r d)$

- Relação dos tempos de término entre mixers e destilação.

$E(i, r m)=E(i, r d)$

- Ordem de descarregamento das parcelas.

$$
S(i 0, r p) \geq E(n, r p-1)
$$

- A destilação deve operar continuamente. 
$\sum_{i}(E(i, r d)-S(i, r d))=H$

- O tempo de preparação deve ser respeitado se um tanque receber uma carga.

$S(i, r t) \geq E(i-1, r t)+T R *(X T(i-1, r t)-X T(i, r t))$

Balanços de massa:

- Balanço material por cru dos recursos em cada slot de tempo.

$L c(i, r, c)=L c(i-1, r, c)-\sum_{r \in \text { out }(r, r 1)} V c(i, r, r 1, c)+\sum_{r \in \text { in }(r, r 1)} V c(i, r, r 1, c)$

- A soma de todos os tipos de crus deve ser equivalente ao volume total.

$L t(i, r)=\sum_{c} L c(i, r, c)$

- A soma da transferência de todos os tipos de crus deve ser equivalente ao volume total transferido.

$V t(i, r, r 1)=\sum_{c} V c(i, r, r 1, c)$

Vazões e limite de capacidade:

- Volume envolvido em uma transferência.

$V T x(r, r 1) * Z(i, r, r 1) \leq V t(i, r, r 1) \leq V T x(r, r 1) * Z(i, r, r 1)$

Qualidade e eliminação de discrepância na composição: Considere $\delta$ com sendo densidade

- Valor máximo imposto para uma propriedade em base mássica.

$$
\begin{aligned}
& \operatorname{PPn}(k) * \sum_{c} V c(i, r m, r d, c) * \delta \leq \sum_{c} V c(i, r m, r d, c) * P P(c, k) * \delta \\
& \leq \operatorname{PPx}(k) * \sum_{c} V c(i, r m, r d, c) * \delta
\end{aligned}
$$

- Eliminação da discrepância de composição.

$$
L c(i, r t, c) * V t(i, r t, r m)=L t(i, r t) * V c(i, r t, r m, c)
$$

\section{RESULTADOS}

É possível observar na Figura 2 que, como esperado, todas as restrições operacionais modeladas foram atendidas, a mais evidente é a de que as destilações devem operar ininterruptamente. Dentro deste cenário conseguimos gerar um lucro de 19103,28 u.m., dentro de um tempo computacional curto, de 1,93 segundos. 
Figura 1 - Gráfico de Gantt do abastecimento de cru

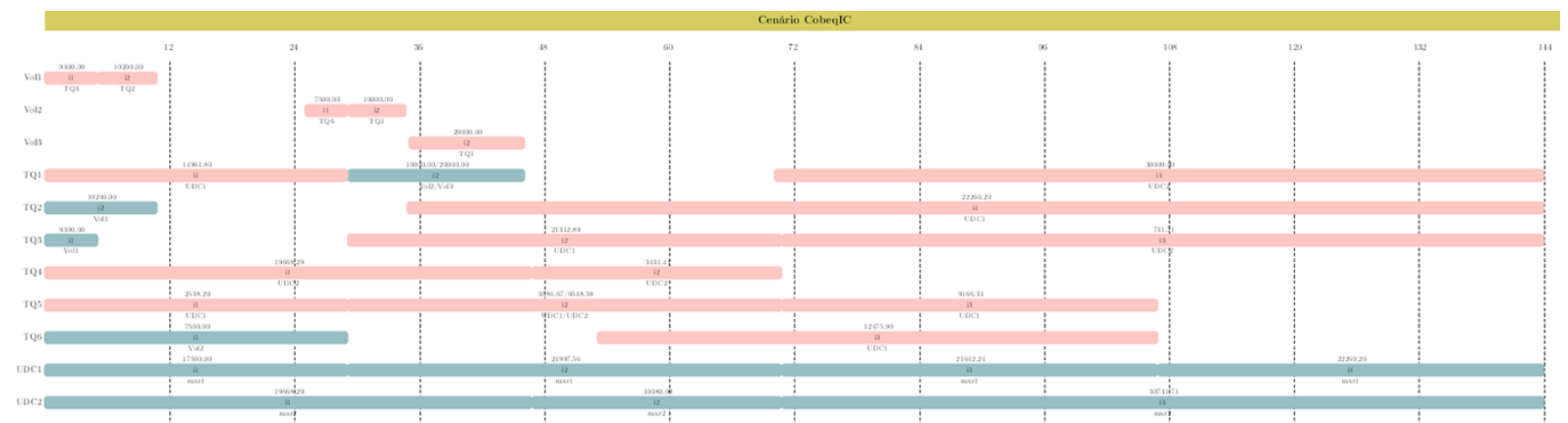

\section{CONCLUSÃO}

Pode-se concluir que os métodos de otimização da programação da produção podem ajudar as indústrias a obter maior ganho e se utilizando do mínimo de recursos, gerando não somente ganhos financeiros, mas muitas vezes ambientais (como utilização de menos água ou minimização da emissão de poluentes).

Devem-se continuar os estudos na área para gerarmos modelos o mais próximos da realidade o possível, dentro das limitações que a matemática e a capacidade computacional permitirem, pois ela tem capacidade que melhorar muito o consumo além de melhorar os resultados gerados por esse consumo.

\section{REFERENCIAS}

Chen, X.; Grossmann, I. \& Zheng, L. (2012). A comparative study of continuous-time models for scheduling of crude oil operations in inland refineries. Computers and Chemical Engineering.

Hu, Y., \& Zhu, Y. (2007). An asynchronous time slotbased continuous time formulation approach for crude oil scheduling. Computers and Applied Chemistry(Em Chinês).

Ierapetritou, M. \& Floudas, C. (1998). Effective Continuous-Time Formulation for ShortTerm Scheduling. Industrial Engineering and Chemistry Research.

Jia, Z., \& Iearapetritou, M. (2003). Mixed-integer linear programming for gasoline blending and distribution scheduling. Industrial Engineering and Chemistry Research.

Pinedo, M. \& Chao, X. (1999). Operations scheduling with applications in manufacturing and services. McGraw Hill. 